\title{
Combined Measurement of Inclusive ep Scattering Cross Sections at HERA
}

\author{
Katarzyna Helena Wichmann* \\ DESY \\ E-mail: katarzyna.wichmannedesy.de
}

\begin{abstract}
A combination is presented of all inclusive deep inelastic cross sections previously published by the $\mathrm{H} 1$ and ZEUS collaborations at HERA for neutral and charged current $e^{ \pm} p$ scattering with zero beam polarisation. The data were taken at proton beam energies of 920, 820, 575 and $460 \mathrm{GeV}$ and an electron beam energy of $27.5 \mathrm{GeV}$. The data correspond to an integrated luminosity of about $1 \mathrm{fb}^{-1}$ and span six orders of magnitude in negative four-momentum-transfer squared, $Q^{2}$, and Bjorken $x$. The correlations of the systematic uncertainties were evaluated and taken into account for the combination. An extraction of $x F_{3}^{\gamma Z}$ and results on electroweak unification and scaling violations are also presented. The high-precision deep inelastic scattering cross sections presented here form a coherent set and they are a major legacy of HERA.
\end{abstract}

XXIII International Workshop on Deep-Inelastic Scattering,

27 April - May 12015

Dallas, Texas

\footnotetext{
* Speaker.
} 


\section{Introduction}

Deep inelastic scattering (DIS) of electrons ${ }^{1}$ on protons at centre-of-mass energies of up to $\sqrt{s} \simeq 320 \mathrm{GeV}$ at HERA has been central to the exploration of proton structure and quark-gluon dynamics as described by perturbative Quantum Chromo Dynamics (pQCD) [1]. This enabled the two collaborations, $\mathrm{H} 1$ and ZEUS, to explore a large phase space in Bjorken $x, x_{\mathrm{Bj}}$, and negative four-momentum-transfer squared, $Q^{2}$. Cross sections for neutral current (NC) interactions have been published for $0.045 \leq Q^{2} \leq 50000 \mathrm{GeV}^{2}$ and $6 \cdot 10^{-7} \leq x_{\mathrm{Bj}} \leq 0.65$ at values of the inelasticity, $y=Q^{2} /\left(s x_{\mathrm{Bj}}\right)$, between 0.005 and 0.95 . Cross sections for charged current $(\mathrm{CC})$ interactions have been published for $200 \leq Q^{2} \leq 50000 \mathrm{GeV}^{2}$ and $1.3 \cdot 10^{-2} \leq x_{\mathrm{Bj}} \leq 0.40$ at values of $y$ between 0.037 and 0.76 . The $\mathrm{H} 1$ and ZEUS collaborations collected total integrated luminosities of approximately $500 \mathrm{pb}^{-1}$ each, divided about equally between $e^{+} p$ and $e^{-} p$ scattering. The results presented here are the final combination of all published H1 and ZEUS measurements from HERA on inclusive DIS in NC and CC reactions [⿰冫欠 $E_{p}=920,820,575$ and $460 \mathrm{GeV}$ corresponding to $\sqrt{s} \simeq 320,300,251$ and $225 \mathrm{GeV}$. They supersede the previous HERA I combined measurement [3]. The combined cross sections were input to QCD analyses at leading order (LO), next-to-leading order (NLO) and at next-to-next-to-leading order (NNLO), providing a new set of parton distribution functions HERAPDF2.0. The QCD fits are not discussed here, please see other contributions and the final publication [2] for details.

\section{Combination of the inclusive cross sections}

In order to combine the published cross sections from the final data sets, they were translated onto common grids and averaged.

The data were taken with several $E_{p}$ values and the double-differential cross sections were published by the two experiments for different reference $\sqrt{s}$ and $\left(x_{\mathrm{Bj}}, Q^{2}\right)$ grids. In order to average a set of data points, the points had to be translated to common $\sqrt{s_{\text {com }}}$ values and common $\left(x_{\mathrm{Bj}, \text { grid }}, Q_{\text {grid }}^{2}\right)$ grids. Four values of $\sqrt{s}_{\text {com }}$ were used: $318 \mathrm{GeV}, 300 \mathrm{GeV} 251 \mathrm{GeV}$ and 225 $\mathrm{GeV}$. Up to 10 data sets were available for a given process. The vast majority of grid points accumulated data from both $\mathrm{H} 1$ and ZEUS measurements; the typical case is six measurements from six different data sets.

For the translation of the cross-section values, predictions for the ratios of the double-differential cross section at the $\left(x_{\mathrm{Bj}}, Q^{2}\right)$ and $\sqrt{s}$ where the measurements took place, and the $\left(x_{\mathrm{Bj}, \text { grid }}, Q_{\text {grid }}^{2}\right)$ to which they were translated, were needed. These predictions were obtained from the data themselves by performing fits to the data using the HERAFitter [ [ [ 5] tool. For $Q^{2} \geq 3 \mathrm{GeV}^{2}$, a NLO QCD fit using the DGLAP formalism was performed. For $Q^{2}<3 \mathrm{GeV}^{2}$, the fit to the fractal model [6, 团] was used.

\subsection{Averaging cross sections}

The original double-differential cross-section measurements were published with their statistical and systematic uncertainties. The systematic uncertainties were classified as either pointto-point correlated or point-to-point uncorrelated. For each data set, all uncorrelated systematic

\footnotetext{
${ }^{1}$ In this paper, the word "electron" refers to both electrons and positrons, unless otherwise stated.
} 
uncertainties were added in quadrature before averaging. Correlated systematic uncertainties were kept separately.

The averaging of the data points was performed using the HERAverager [8] tool which is based on a $\chi^{2}$ minimisation method [6]. This method imposes that there is one and only one correct value for the cross section of each process at each point of the phase space. These values are estimated by optimising a vector, $m$, which is the result of the averaging for the cross sections. The $\chi^{2}$ definition used takes into account the correlated and uncorrelated systematic uncertainties of the $\mathrm{H} 1$ and ZEUS cross-section measurements and allows for shifts of the data to accommodate the correlated uncertainties. For a single data set, $d s$, the $\chi^{2}$ is defined as

$$
\chi_{\exp , d s}^{2}(m, b)=\sum_{i}^{d s}+\sum_{j}^{b}=\sum_{i} \frac{\left[m^{i}-\sum_{j} \gamma_{j}^{j, d s} m^{i} b_{j}-\mu^{i, d s}\right]^{2}}{\delta_{i, d s, \text { stat }}^{2} \mu^{i, d s}\left(m^{i}-\sum_{j} \gamma_{j}^{i, d s} m^{i} b_{j}\right)+\left(\delta_{i, d s, \text { uncor }} m^{i}\right)^{2}}+\sum_{j} b_{j}^{2},
$$

where $\mu^{i, d s}$ is the measured value at the point $i$ and $\gamma_{j}^{i, d s}, \delta_{i, d s, \text { stat }}$ and $\delta_{i, d s \text {,uncor }}$ are the relative correlated systematic, relative statistical and relative uncorrelated systematic uncertainties, respectively. For the reduced cross-section measurements, $\mu^{i, d s}=\sigma_{r}^{i, d s}, i$ runs over all points on the $\left(x_{\mathrm{Bj}, \text { grid }}, Q_{\mathrm{grid}}^{2}\right)$ plane for which a measurement exists in $d s$. The components $b_{j}$ of the vector $b$ represent correlated shifts of the cross sections in units of sigma of the respective correlated systematic uncertainties; the summations over $j$ extend over all correlated systematic uncertainties.

All the NC and CC cross-section data from $\mathrm{H} 1$ and ZEUS are combined in one simultaneous minimisation. Therefore, the resulting shifts of the correlated systematic uncertainties propagate coherently to both NC and CC data. The 2927 published cross section were combined to become 1307 combined cross-section measurements. For the resulting 1620 degrees of freedom, a $\chi^{2}=$ 1687 was obtained, indicating good consistency of all data. More details on the data combination can be found in the final publication [2].

\section{Combined inclusive $e^{ \pm} p$ cross sections}

The combined reduced cross sections for NC and CC ep scattering together with their statistical, uncorrelated and total correlated systematic uncertainties are tabulated in the final publication [2]. The new values supersede those published previously [3]. The total uncertainties are below $1.5 \%$ over the $Q^{2}$ range of $3 \leq Q^{2} \leq 500 \mathrm{GeV}^{2}$ and below $3 \%$ up to $Q^{2}=3000 \mathrm{GeV}^{2}$.

In Fig. 1, the individual and the combined reduced cross sections for $\mathrm{NC} e^{+} p$ DIS scattering are shown as a function of $Q^{2}$ for selected values of $x_{\mathrm{Bj}}$. The improvement due to combination is clearly visible. The improvement in comparison to the previous HERA I combination [3] is especially significant at high $Q^{2}$. As the integrated luminosity for $e^{-} p$ scattering was very limited for the HERA I period, the improvements due to the new combination are even more substantial than for $e^{+} p$ scattering. This is clearly visible in Fig. Z, where a comparison between the new combination and the combination of HERA I data for $e^{-} p$ DIS is shown.

The high-precision NC and CC DIS cross sections for various $\sqrt{s}_{\text {com }}$ presented here form a coherent set spanning six orders of magnitude in $Q^{2}$ and $x_{\mathrm{Bj}}$. As an example the NC cross sections with lowest $Q^{2}$ and the CC cross sections with highest $Q^{2}$ are shown in Figures 3 and 4 , respectively, compared to NNLO HERAPDF2.0. These measurements are a major legacy of HERA. 


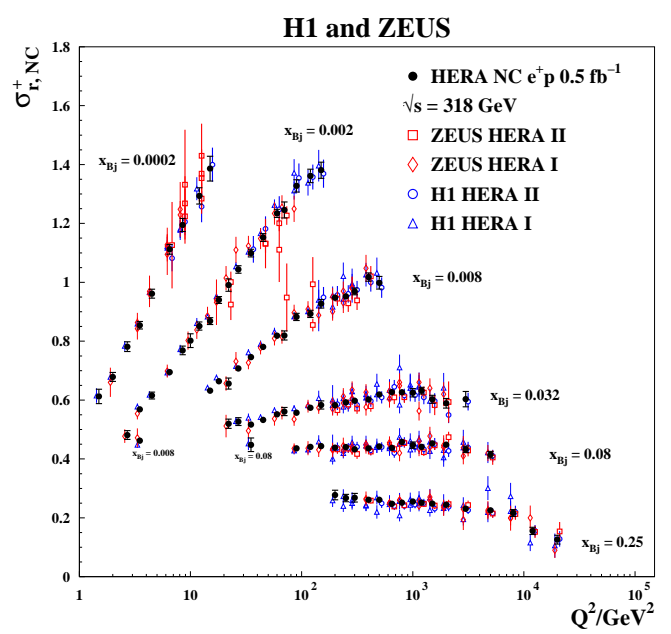

Figure 1: The combined HERA data for the inclusive NC $e^{+} p$ reduced cross sections as a function of $Q^{2}$ for six selected values of $x_{\mathrm{Bj}}$ compared to the individual $\mathrm{H} 1$ and ZEUS data. The individual measurements are displaced horizontally for better visibility. Error bars represent the total uncertainties.

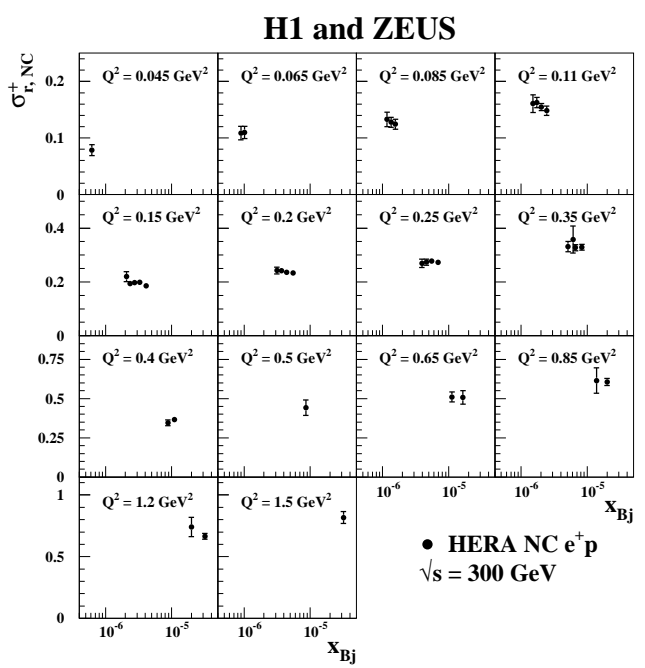

Figure 3: The combined HERA data for the inclusive $\mathrm{NC} e^{+} p$ reduced cross sections at $\sqrt{s}=300 \mathrm{GeV}$ at very low $Q^{2}$. Error bars represent the total uncertainties.

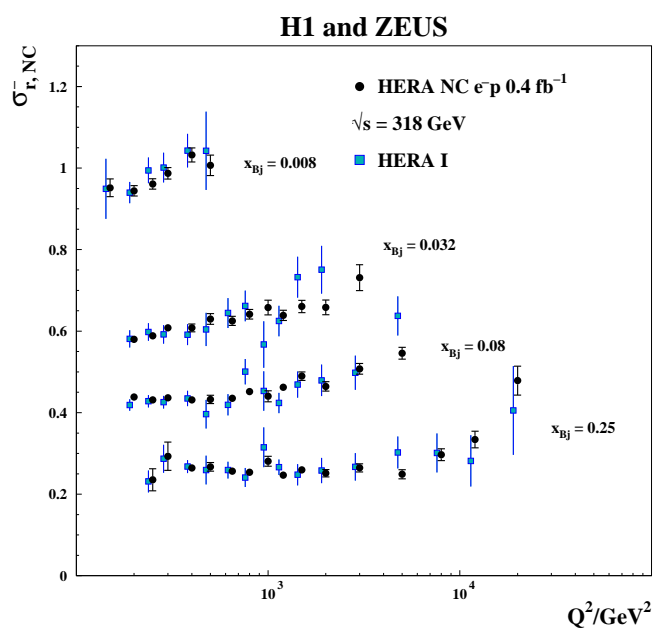

Figure 2: The combined HERA data for the inclusive NC $e^{-} p$ reduced cross section as a function of $Q^{2}$ for four selected values of $x_{\mathrm{Bj}}$ compared to the results from HERA I alone [3]. The two measurements are displaced horizontally for better visibility. Error bars represent the total uncertainties.

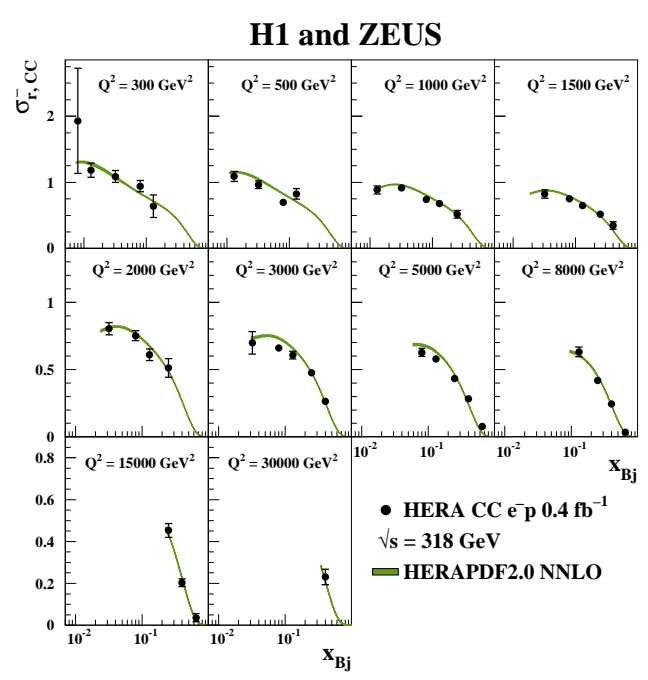

Figure 4: The combined HERA CC $e^{-} p$ reduced cross sections at $\sqrt{s}=318 \mathrm{GeV}$ with overlaid predictions from HERAPDF2.0 NNLO. The bands represent the total uncertainties on the predictions.

\section{Electroweak effects and scaling violations}

The precise data and the predictions from HERAPDF2.0 were used to examine both electroweak effects and QCD effects. 


\subsection{Electroweak unification and the structure function $x F_{3}^{\gamma Z}$}

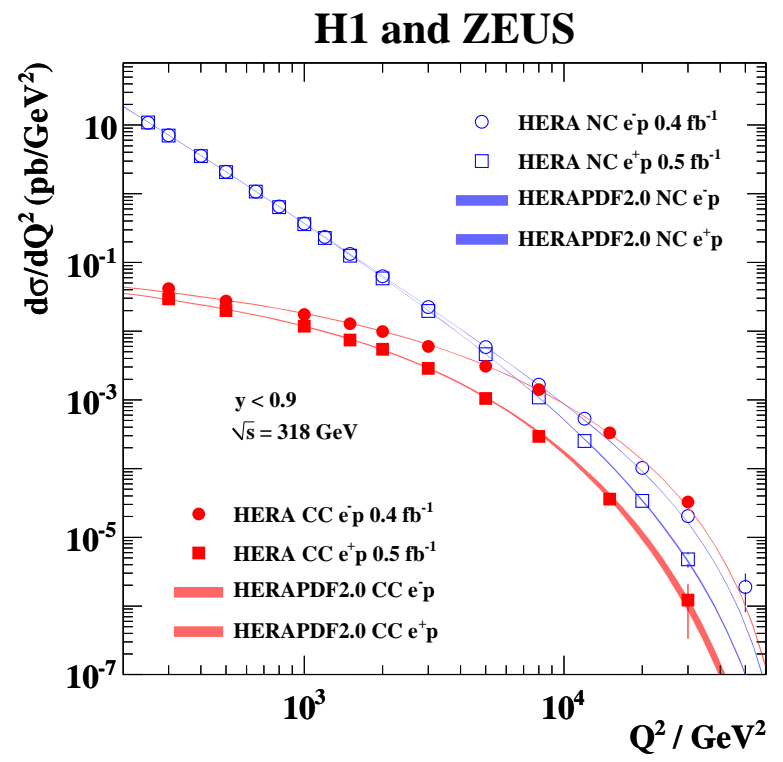

Figure 5: The combined HERA NC and $\mathrm{CC} e^{-} p$ and $e^{+} p$ cross sections, $\mathrm{d} \sigma / \mathrm{d} Q^{2}$, together with predictions from HERAPDF2.0 NLO. The bands represent the total uncertainty on the predictions.

bosons, demonstrating the success of electroweak unification in the Standard Model with impressive precision.

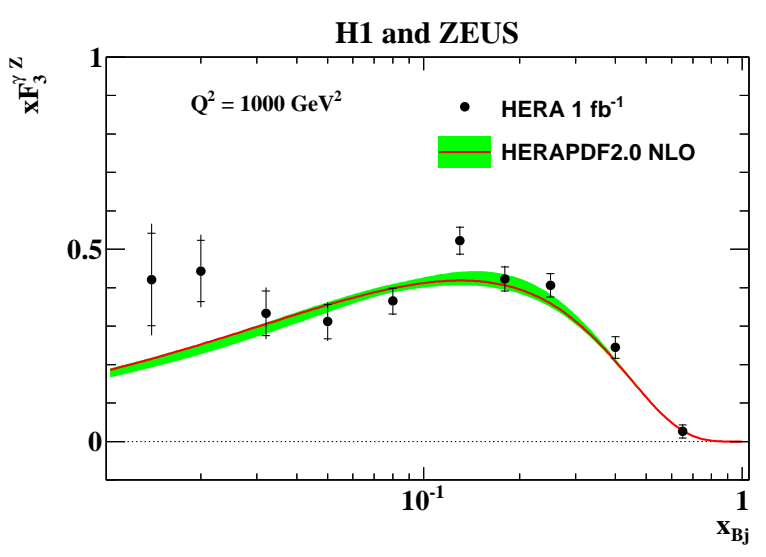

Figure 6: The structure function $x F_{3}^{\gamma Z}$ averaged over $Q^{2} \geq$ $1000 \mathrm{GeV}^{2}$ at the scale $Q^{2}=1000 \mathrm{GeV}^{2}$ together with the prediction from HERAPDF2.0 NLO. The band represents the total uncertainty on the prediction.
The combined reduced cross sections were integrated to obtain the differential cross sections $\mathrm{d} \sigma / \mathrm{d} Q^{2}$. The integration over $x_{\mathrm{Bj}}$ of the doubledifferential cross-sections $\mathrm{d}^{2} \sigma / \mathrm{d} Q^{2} \mathrm{~d} x_{\mathrm{Bj}}$ was performed in the region $0<$ $y<0.9$, using the shapes as predicted by HERAPDF2.0 NLO. All correlated and uncorrelated uncertainties were taken into account. The crosssections $\mathrm{d} \sigma / \mathrm{d} Q^{2}$ are shown in Fig. 5 for $\mathrm{NC}$ and $\mathrm{CC} e^{-} p$ and $e^{+} p$ scattering together with predictions from HERAPDF2.0 NLO. Whereas the NC cross sections are three orders of magnitude larger at low $Q^{2} \approx 100 \mathrm{GeV}^{2}$, where they are dominated by virtual photon exchange, the $\mathrm{NC}$ and $\mathrm{CC}$ cross sections become similar in magnitude at $Q^{2} \approx 10000 \mathrm{GeV}^{2}$, i.e. at around the mass-scale squared of the electroweak

The data also clearly demonstrate that the $\mathrm{NC} e^{-} p$ and $\mathrm{NC} e^{+} p$ cross sections are the same when photon exchange is dominant but they start to differ at $Q^{2} \approx 10000 \mathrm{GeV}^{2}$ when $\gamma-Z$ interference becomes important. The

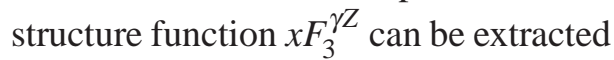
by subtracting the $\mathrm{NC} e^{+} p$ from the $\mathrm{NC} e^{-} p$ cross sections measured as a function of $x_{\mathrm{Bj}}$ in bins of $Q^{2}$. This directly probes the valence structure of the proton. The valence-quark distributions and hence $x F_{3}^{\gamma Z}$ depend only minimally on the scale, i.e. only small corrections are needed to translate all values of $x F_{3}^{\gamma Z}$ to a common scale of $1000 \mathrm{GeV}^{2}$. This was done using HERAPDF2.0 NLO. The translation factors 
were close to unity for most points. The translated $x F_{3}^{\gamma Z}$ values were averaged using the method described in Section 2. A full covariance matrix was built using the information on the individual sources of uncertainty. The averaging of the $x F_{3}^{\gamma Z}$ values has a $\chi^{2} /$ d.o.f. $=58.8 / 57$ demonstrating the consistency of the data for different values of $Q^{2}$. The result is presented in Fig. 6 together with the prediction of HERAPDF2.0 NLO. The data are well described by HERAPDF2.0 NLO.

\subsection{Helicity effects in $\mathrm{CC}$ interactions}

Figure 7 presents the reduced cross sections $\sigma_{r, \mathrm{CC}}^{+}$and $\sigma_{r, \mathrm{CC}}^{-}$for CC inclusive $e^{+} p$ and $e^{-} p$ scattering, respectively, compared to HERAPDF2.0 NNLO. In the Quark Parton Model the CC cross sections are related to the quark densities in the proton as follows

$$
\sigma_{r, \mathrm{CC}}^{+} \approx\left(x \bar{U}+(1-y)^{2} x D\right), \quad \sigma_{r, \mathrm{CC}}^{-} \approx\left(x U+(1-y)^{2} x \bar{D}\right),
$$

where the terms $x U, x D, x \bar{U}$ and $x \bar{D}$ denote the sums of parton distributions for up-type and downtype quarks and anti-quarks, respectively. The $e^{+} p$ cross sections are affected strongly by the helicity factor $(1-y)^{2}$. Therefore, the contribution of the valence quarks is suppressed at high $y$ which translates to high $Q^{2}$ for fixed $x_{\mathrm{Bj}}$. The $e^{-} p$ cross section is almost unaffected, because the helicity factor applies to the anti-quarks which as part of the sea are already suppressed at high $x_{\mathrm{Bj}}$.
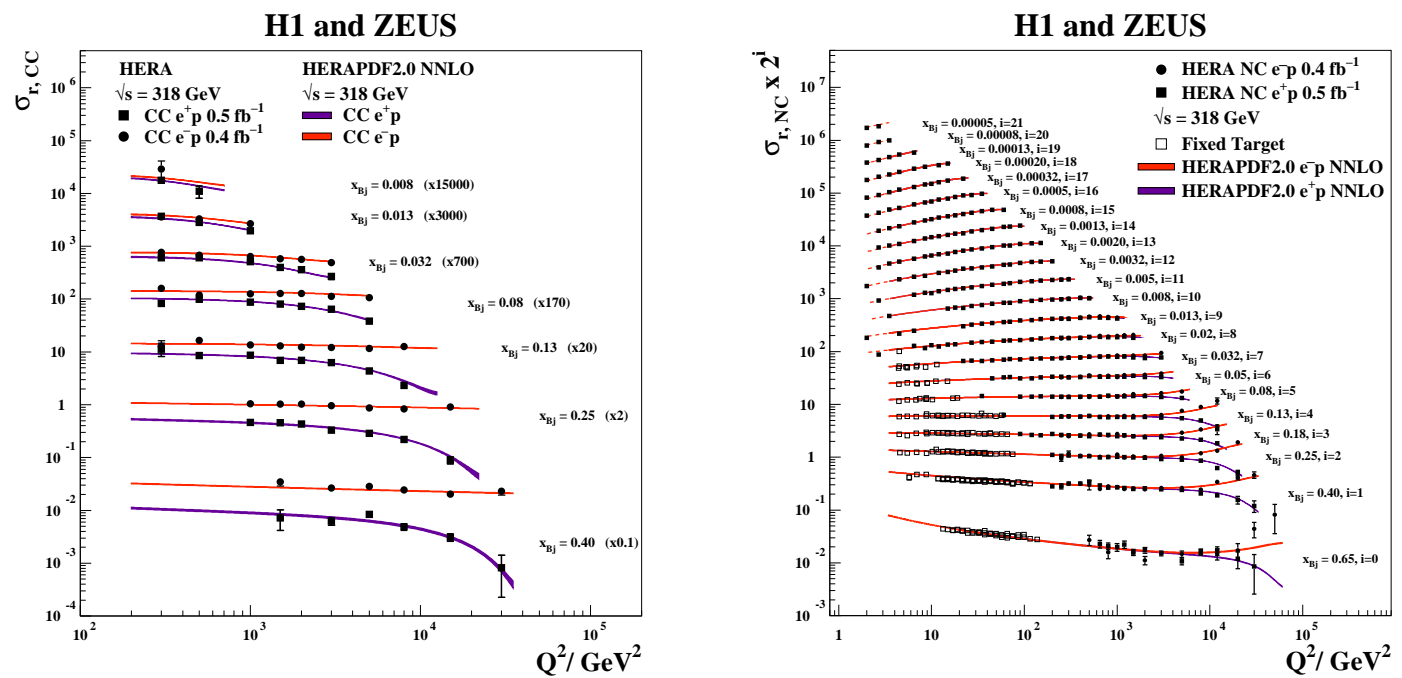

Figure 7: The combined HERA data for inclu- Figure 8: The combined HERA data for the inclusive $\mathrm{CC} e^{+} p$ and $e^{-} p$ reduced cross sections sive $\mathrm{NC} e^{+} p$ and $e^{-} p$ reduced cross sections together at $\sqrt{s}=318 \mathrm{GeV}$ with overlaid predictions of with fixed-target data [9, 10] and the predictions of HERAPDF2.0 NLO. The bands represent the total HERAPDF2.0 NNLO. The bands represent the touncertainties on the predictions. tal uncertainties on the predictions. Dashed lines indicate extrapolation into kinematic regions not included in the fit.

\subsection{Scaling violations}

Scaling violations, i.e. the dependence of the structure functions on $Q^{2}$ at fixed $x_{\mathrm{Bj}}$, are related to the gluon content of the proton. A large kinematic range is required to demonstrate these viola- 
tions clearly. Figure 8 show the inclusive $\mathrm{NC} e^{+} p$ and $e^{-} p$ HERA data together with fixed-target data [9, 10] and the predictions of HERAPDF2.0 NNLO. The data presented span more than four orders of magnitude, both in $Q^{2}$ and $x_{\mathrm{Bj}}$. The scaling violations are clearly visible over this large kinematic range. They are well described by HERAPDF2.0, both at NLO and NNLO.

\section{Summary}

The H1 and ZEUS collaborations measured inclusive $e^{ \pm} p$ scattering cross sections at HERA from 1994 to 2007, collecting a total integrated luminosity of about $1 \mathrm{fb}^{-1}$. The data were taken in two different beam configurations, called HERA I and HERA II, at four different centre-of-mass energies and with two different detectors changing and improving over time. All inclusive data were combined to create one consistent set of $\mathrm{NC}$ and $\mathrm{CC}$ cross-section measurements for unpolarised $e^{ \pm} p$ scattering, spanning six orders of magnitude in both negative four-momentum-transfer squared, $Q^{2}$, and Bjorken $x$. The data from many measurements made independently by the two collaborations proved to be consistent with a $\chi^{2}$ per degree of freedom being 1.04 for the combination. The combined cross sections are the most precise measurements ever published for $e p$ scattering over such a large kinematic range. They are provided for values of $Q^{2}$ between $Q^{2}=0.045 \mathrm{GeV}^{2}$ and $Q^{2}=50000 \mathrm{GeV}^{2}$ and values of $x_{\mathrm{Bj}}$ between $x_{\mathrm{Bj}}=6 \times 10^{-7}$ and $x_{\mathrm{Bj}}=0.65$. The precision of the data were exploited to demonstrate electroweak unification and extract $x F_{3}^{\gamma Z}$ above $Q^{2}=1000 \mathrm{GeV}^{2}$. Scaling violation is demonstrated over four orders of magnitude in $Q^{2}$.

\section{References}

[1] A. Cooper-Sarkar and R. Devenish, "Deep inelastic Scattering", Oxford Univ. Press (2011), ISBN 978-0-19-960225-4.

[2] H. Abramowicz et al., [H1 and ZEUS Collaborations], Combination of Measurements of Inclusive Deep Inelastic $e^{ \pm} p$ Scattering Cross Sections and QCD Analysis of HERA Data, DESY-15-039 (2015), submitted to EPJS, arXiv:1506.06042.

[3] F. D. Aaron et al., [H1 and ZEUS Collaborations], Combined Measurement and QCD Analysis of the Inclusive $e^{ \pm}$p Scattering Cross Sections at HERA, 10.1007/JHEP01(2010)109 [arXiv:0911.0884].

[4] S. Alekhin et al., HERAFitter, Open Source QCD Fit Project, arXiv:1410.4412.

[5] The documentation and the package can be found at: www.herafitter.org

[6] F. D. Aaron et al., [H1 Collaboration], Measurement of the Inclusive ep Scattering Cross Section at Low $Q^{2}$ and $x$ at HERA, Eur. Phys. J. C 63, 625 (2009) [arXiv:0904.0929].

[7] T. Lastovicka, Selfsimilar properties of the proton structure at low x, Eur. Phys. J. C 24, 529 (2002) [hep-ph/0203260].

[8] The documentation and the package can be found at: https://wiki-zeuthen.desy.de/HERAverager

[9] A. C. Benvenuti et al., [BCDMS Collaboration], A High Statistics Measurement of the Proton Structure Functions $F(2)\left(x, Q^{2}\right)$ and $R$ from Deep Inelastic Muon Scattering at High $Q^{2}$, Phys. Lett. B 223, 485 (1989).

[10] M. Arneodo et al., [New Muon Collaboration], it Measurement of the proton and deuteron structure functions, F2(p) and F2(d), and of the ratio sigma-L / sigma-T, Nucl. Phys. B 483, 3 (1997). 